Review

\title{
Hyperbaric Oxygen Therapy in Liver Diseases
}

\author{
Yun Sun ${ }^{1,2 *}$, Yankai Wen ${ }^{3,4 *}$, Chanjuan Shen ${ }^{1,2 *}$, Yuanrun Zhu ${ }^{5}$, Wendong You ${ }^{5}$, Yuanyuan Meng6, Lijuan \\ Chen ${ }^{1,2}$, Yiping Feng ${ }^{1,2}$, Xiaofeng Yang ${ }^{5}$, Zuo-Bing Chen ${ }^{1,2}$ \\ 1. Department of Rehabilitation Medicine, The First Affiliated Hospital, Zhejiang University, Hangzhou, China. \\ 2. Department of Hyperbaric Oxygen, The First Affiliated Hospital, Zhejiang University, Hangzhou, China. \\ 3. School of Biomedical Engineering, Shanghai Jiao Tong University, Shanghai, China. \\ 4. Department of Liver Surgery, Renji Hospital, School of Medicine, Shanghai Jiao Tong University, Shanghai, China. \\ 5. Department of Neurosurgery, The First Affiliated Hospital, Zhejiang University, Hangzhou, China. \\ 6. Department of Pediatric Medicine, The Children's Hospital, Zhejiang University, Hangzhou, China. \\ * These authors contribute equally to this work. \\ $\triangle$ Corresponding author: Zuo-Bing Chen, MD, Department of Rehabilitation Medicine, The First Affiliated Hospital, Zhejiang University, 79 Qingchun Road, \\ Hangzhou, China. 310000, Telephone: +86 13957116610, E-mail: czb1971@zju.edu.cn \\ (C) Ivyspring International Publisher. This is an open access article distributed under the terms of the Creative Commons Attribution (CC BY-NC) license \\ (https://creativecommons.org/licenses/by-nc/4.0/). See http://ivyspring.com/terms for full terms and conditions.
}

Received: 2018.01.05; Accepted: 2018.04.12; Published: 2018.05.22

\begin{abstract}
Hyperbaric oxygen therapy (HBOT) is an efficient therapeutic option to improve progress of lots of diseases especially hypoxia-related injuries, and has been clinically established as a wide-used therapy for patients with carbon monoxide poisoning, decompression sickness, arterial gas embolism, problematic wound, and so on. In the liver, most studies positively evaluated HBOT as a potential therapeutic option for liver transplantation, acute liver injury, nonalcoholic steatohepatitis, fibrosis and cancer, especially for hepatic artery thrombosis. This might mainly attribute to the anti-oxidation and anti-inflammation of HBOT. However, some controversies are existed, possibly due to hyperbaric oxygen toxicity. This review summarizes the current understandings of the role of HBOT in liver diseases and hepatic regeneration. Future understanding of HBOT in clinical trials and its in-depth mechanisms may contribute to the development of this novel adjuvant strategy for clinical therapy of liver diseases.
\end{abstract}

Key words: hyperbaric oxygen; liver diseases; toxicity

\section{Introduction}

Hyperbaric oxygen therapy (HBOT) was defined as "an intervention in which an individual breathes near $100 \%$ oxygen intermittently while inside a hyperbaric chamber that is pressurized to greater than sea level pressure (one atmosphere absolute [ATA])" by the Undersea and Hyperbaric Medicine Society [1]. In recent decades, numerous studies supported HBOT as an efficient therapeutic option to improve progress of lots of diseases especially hypoxia-related injuries. Furthermore, HBOT has been clinically established as a wide-used therapy for patients with carbon monoxide poisoning, decompression sickness, arterial gas embolism, problematic wound, and so on; and HBOT was also an important adjunctive therapy to treat diseases accompanied by impaired oxygen delivery [2]. In the liver, HBOT has been studied in hepatic artery thrombosis (HAT), acute liver injury, nonalcoholic steatohepatitis (NASH), bacterial infection, fibrosis and liver-related cancer. In this review, we will summarize the role of HBOT in liver diseases and hepatic regeneration, discuss hyperbaric oxygen toxicity, and evaluate the potential of clinical application of HBOT in liver.

\section{HBOT in acute liver injury}

Liver transplantation is the definitive treatment in end-stage liver diseases, which includes selected liver malignancies and liver failure [3]. However, at present, liver ischemia, preservation and reperfusion injury (IPRI) is still the major problem and hotspot for researchers. Over past decades, numerous studies seemed to support the application of HBOT in IPRI [4, 5]. In 1967, Macdougal, J.D. et al. [6] reported that in vitro cultures of adult rat liver required a higher 
oxygen tension for maintenance, which indicated that hyperbaric oxygen might improve injury of organ preservation. Furthermore, Slapak, M. et al. [7] and Spilg, H. et al. [8] successfully preserved animal livers for 24 hours under three atmospheres of oxygen and hypothermia. Another in vitro study showed that HBOT during liver cold storage protected against subsequent hepatic reperfusion injury by attenuating oxidant stress and the depletion of energy loss, such as ATP [9]. In vivo animal models were subjected to determine the effect of HBOT in IPRI since then. Prolonged preservation was found to enhance IPRI post-orthotopic liver transplantation (OLT) in rats, characterized by more necrosis and apoptosis [10]. And similar to the in vitro study [9], HBOT reduced the severity of IPRI by protecting hepatocytes from necrosis and apoptosis and improving sinusoidal diameter and microvascular density index in the rat model of OLT [10].

The majority of in vivo animal studies evaluating HBOT used a model of hepatic warm ischemiareperfusion (I/R) injury. HBOT after surgery was shown to improve rat hepatic $I / R$ injury via suppressing the accumulation of neutrophils. However, it is of importance to emphasize that only early HBOT given before neutrophil-mediated injury phase (in 3 hours after reperfusion) might take protective effect [11]. In addition, early HBOT after hepatic I/R injury also improved mitochondrial functions [12]. Besides, the therapeutic effect of HBOT in different periods of $I / R$ was determined. And studies showed that HBOT subjected to early during ischemia played a more favorable effect compared to be subjected to both periods of ischemia and reperfusion; otherwise, HBOT given in the period of reperfusion was associated with a more severe hepatocyte apoptosis and liver damage [13, 14]. Yet the protection of HBOT in hepatic I/ $R$ injury still remains controversial. Margarido, M. R. et al. [15] reported that HBOT during the ischemic period did not prevent the installation of mitochondrial edema induced by hepatic I/R. Moreover, HBOT preconditioning gains more controversies. Hyperbaric oxygen administration one day before $I / R$ protected rat liver against subsequent $I / R$ injury, and administration three days before $I / R$ took no effects [16]. Several studies also supported that injury induced by $I / R$ immediately after a short-time HBOT (less than 60 minutes) was more alleviated than $I / R$ without HBOT, and this protection might mediate by HO-1 and be characterized by attenuated hepatocyte apoptosis and improvement of mitochondrial functions [17-20]. However, a 120-minute HBOT immediately before I/R surgery did not protect against injury, with higher liver malondialdehyde
(MDA) and nitric oxide (NO) levels compared to the 60-minute HBOT [18]. Besides, two studies showed that HBOT preconditioning for 60 and 90 minutes even aggravated liver $I / R$ injury via enhanced neutrophil accumulation $[15,21]$. No effects on mitochondrial functions by a 60-minutes HBOT preconditioning were also reported [22].

Although discrepancies of effects of HBOT exist, the protective role of early HBOT after $I / R$ is confirmed by animal studies. Thus, early HBOT (in 3 hours) in patients receiving liver transplantation might improve their prognosis, and further related clinical trials are necessary.

HBOT has also been reported as an adjuvant treatment to successfully rescue a 3-year-old girl with acute liver failure (ALF) and coma [23], which suggests the protective role of HBOT in acute liver injury. Coincidentally, HBOT helped to rescue a 28-year-old man ingested a large dose of carbon tetrachloride [24]. HBOT was also shown to protect the liver from hepatotoxicity of halothane anesthesia or its metabolites in patients in some cases [25].

In animal studies, hyperoxia protects hepatocytes against carbon tetrachloride toxicity [26-29]. Immediate HBOT attenuated the rise in lipid peroxidation and necrosis, and prevented the mortality 24 hours after intoxication; otherwise, delayed HBOT (one hour after intoxication) prevented the mortality but was less effective in alleviating necrosis [26]. HBOT could decrease free radical formation in carbon tetrachloride-treated liver [28]. However, loss of this protection was very rapid, because HBOT 6 hours after carbon tetrachloride administration augmented hepatic necrosis instead [29]. Thus, it is better to use HBOT for ALF as early as possible.

In zymosan-shocked rats, HBOT attenuated the increase of myeloperoxidase (MPO) and MDA in liver, finally prevented the development of liver failure [30]. HBOT also attenuated the zymosaninduced expression of TLR2 and TLR4, NF-KB activation, and cytokine production in liver, indicating hyperbaric oxygen as an inflammatory regulator [31]. In another ALF model of acetaminophen (APAP)-induced liver injury, HBOT reduced early APAP hepatotoxicity via regulating HIF-1a [32]. And HBOT augmented the protective effect of $\mathrm{N}$-acetylcysteine (NAC) on APAP-induced liver injury [33]. Although nearly all studies reported the protective role of HBOT in acute liver injury, it is noteworthy that prolonged HBOT ( 3 or 4 hours) aggravated hepatic necrosis induced by APAP, bromobenzene, dimethylnitrosamine (DEN) or thioacetamide (TAA), and had no effect on liver injury induced by galactosamine (GalN) or lipopolysaccharide (LPS). This 
prolonged treatment caused a $50 \%$ mortality in rats and hamsters intoxicated with APAP, and this high mortality was not due to APAP-induced hepatotoxicity but rather due to intolerance to long-time hyperbaric oxygen exposure [29]. The effects of HBOT on acute liver injury and ALF need to be further investigated.

\section{HBOT in liver regeneration}

All of studies now published confirmed that HBOT promoted liver regeneration after hepatictomy, including pre-surgery and post-surgery HBOT [34, 35]. In vitro study reported hyperbaric oxygen stimulated hepatocyte proliferation via normalizing Mrp-2 localization to apical membrane and subsequently activating the transporter function [36]. HBOT is closely associated with mitochondrial function improvement in liver regeneration. And HBOT stabilized energy metabolism by inducing oxygen delivery after partial hepatectomy (PHx) [37]. HBOT also decreased MDA and increased antioxidant activities, including glutathione (GSH) and superoxide dismutase (SOD) activities to benefit liver regeneration [38]. Furthermore, biochemical analysis of mitochondrial respiration, respiratory control rate (RCR) and mitochondrial permeability transition (MPT) supported that HBOT protected mitochondria during liver regeneration [39]. Besides, both of presurgery and post-surgery HBOT could promote angiogenesis via enhanced VEGF in regenerating liver $[35,40]$. Ganglioside GM3 was also involved in mediation of beneficial effects of pre-operatively oxygenation after PHx [41]. Similarly, HBOT augmented hepatocyte proliferation in hepatic injuryinduced regeneration [10]. More importantly, HBOT facilitated liver regeneration in living donors after liver transplantation [42]. All of previous studies supported the HBOT in liver regeneration, especially for liver donors after hepatectomy.

\section{HBOT in liver fibrosis}

Few studies of the effects of HBOT on hepatic fibrosis were performed. Using a hepatic injury and fibrosis model of bile duct ligation (BDL), investigators found that HBOT alleviated oxidative injury, hepatocyte damage, bile duct proliferation and fibrosis [43, 44]. The effects of HBOT on fibrotic reduction seems to be due to the its antioxidant role against injury [43]. However, we still have a long way to understand the definite effect of HBOT on liver fibrosis and cirrhosis.

\section{HBOT in nonalcoholic steatohepatitis}

Using monosodium glutamate (MSG) mice, which are an animal model of NASH, obesity, diabetes and hyperlipidemia, Tsuneyama, K. et al. [45] found HBOT significantly lowered the body weight, but increased oxidative stress and augmented cellular damage and TNFa expression. Thus, HBOT might not a fine option for NASH, and more investigations are necessary.

\section{HBOT in liver-related cancer}

As tumor hypoxia is important for cancer progression, HBOT could be used as a candidate therapy. Yet hyperbaric oxygen promotes cell proliferation, and no one could confirm that HBOT does not promote cancer cell proliferation in patients. This issue limits the development of HBOT as an anti-tumor therapy clinically [46]. For hepatocellular carcinoma (HCC), HBOT might overcome deficiencies in systemic and hepatic oxygen supply and subsequently diminish post-operative complications. In addition, HBOT improved postoperative immune response and long-term survival after liver section in HCC patients [47]. Although the benefit of HBOT was determined, the potential risk of HBOT in cancer patients was not evaluated and it could not be ignored. An early animal study demonstrated that HBOT did not have a tumor stimulatory effect on colorectal liver metastases but significantly reduced necrosis and proliferation in tumor compared to the non-HBOT group [48], which suggested that HBOT might potentially be safe and efficient for cancer.

In solid tumors, hypoxia always associated with radiotherapy and chemotherapy resistance. HBOT could increase tissue oxygen pressure and content, and might prevent this resistance. Thus, hyperbaric oxygen combined with radiotherapy or chemotherapy has been intensively investigated [46]. An in vitro study showed that HBOT, sorafenib or cisplatin alone all could inhibit HCC cell growth, yet HBOT combined with sorafenib or cisplatin led to much greater synergistic proliferative inhibition and apoptotic induction [49], suggesting a potential adjuvant application of HBOT in HCC patients.

\section{HBOT in sepsis-induced liver injury}

A quite early study in 1976 reported that daily HBOT begun either 1 day or 1 week after anerobic infection of Fusobacterium necrophorum and Bacteroides fragilis significantly reduced the number and size of liver abscesses in mice [50]. In septic rats, hyperbaric oxygen pretreatment combining $\mathrm{HO}-1$ activation alleviated LPS-induced liver injury, but HBOT alone did not affect liver injury, indicating that HO-1mediated the protective role of HBOT [51]. In Escherichia coli-induced sepsis, HBOT sufficiently augmented the protection of cefepime, and the combined therapy reversed most septic rats to present 
a normal liver histology, though HBOT alone helped nothing for these septic rats [52]. Another protective mechanism of HBOT might be its effective prevention of bacterial translocation [53]. Overall, HBOT is a potential adjuvant option for treatment of sepsis, yet more clinical investigations are necessary.

\section{HBOT in artery thrombosis}

Hepatic artery thrombosis can occur in up to $10 \%$ of adult or children OLT [54-56]. HBOT significantly delayed retransplantation of child patients with HAT [57]. Another case showed that six-time HBOT successfully recanalized hepatic artery in children with HAT, while alteplase failed open the artery before HBOT [55]. And they found the patients tolerated HBOT and suffered no complications from the HBOT $[55,57]$. Similarly, HBOT could also treat adult patients with HAT as reported $[54,56]$. Thus, HBOT would be a promising therapy for patients with HAT because of its efficiency and patient tolerance. Even though some case reports suggested the benefit of the HBOT in HAT, more randomized controlled trials are needed to explore the application of the HBOT for HAT.

\section{Hyperbaric oxygen toxicity}

With limited clinical experience of hyperbaric oxygen application in 1960s, there happened at least one death attribute to the use of hyperbaric oxygen [58]. The hyperbaric oxygen toxicity in liver was

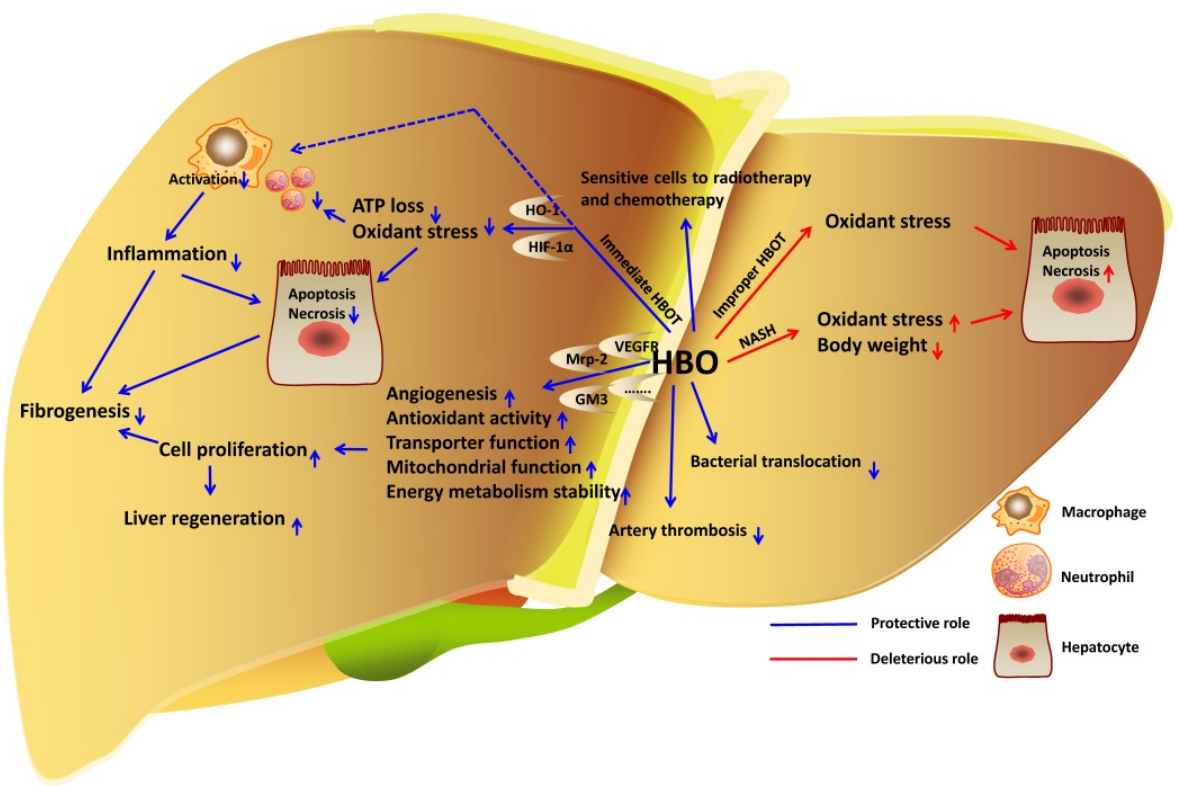

Figure 1. HBOT in liver diseases. In liver, immediate HBOT reduces inflammation and oxidant stress to protect hepatocytes in liver injury. HBOT also facilitates hepatocyte proliferation and improves fibrogenesis. HBOT inhibits bacterial translocation to attenuate sepsis-induced liver injury. In addition, HBOT is definitely an effective therapy for artery thrombosis. Even though the role of $\mathrm{HBO}$ in liver cancer cell proliferation is undetermined, combination of HBOT and radiotherapy or chemotherapy sensitive cancer cells to death. However, improper HBOT leads to increase of oxidant stress and hepatotoxicity. studied since then. Oxygen toxicity was primarily due to initiate the free-radical chain reaction by oxygen, which then aggravated spontaneously with the consequent lipid peroxidation. The early phase of HBOT involved the lipid peroxide metabolism, which could be reduced via glutathione peroxidase reaction. And the latter phase linked to glutathione oxidation and release of GSSG. The GSSG was then reduced via the glutathione reductase reaction, linking to NADPH oxidation, which subsequently generating more reactive oxygen species (ROS), and finally leading to cell death [59, 60]. All of these alterations by HBOT may cause the pathological disruption of physiological functions including interrupted biosynthesis. For instance, exposure of organ cultures of rat livers to hyperbaric oxygen resulted in rapid loss of succinic dehydrogenase and cytochrome oxidase activity [61]. Nutritional antioxidant supplementation may offset HBOT-induced oxidative stress. It has been reported that vitamin $C$, vitamin $E$ and succinate could protect liver against hyperbaric oxygen toxicity [62-64]. Noteworthily, most hyperbaric oxygen toxicity observed was under the condition of more than 3 ATA; but in clinical application, the hyperbaric oxygen therapy is always controlled under 2 ATA. Thus, at present, the case of clinical HBOT-induced injury or death in patients is quite rare. However, the safe dose and frequency of HBOT in liver diseases need to be paid great attention while clinical application.

\section{Conclusion}

Most studies positively evaluated HBOT as a potential adjunct for LT, acute liver injury, NASH, fibrosis and cancer, especially for HAT (Fig. 1). This might mainly attribute to the anti-oxidation and anti-inflammation of HBOT, and HO-1 seems to be closely involved in HBOT-mediated protection. Furthermore, it would have better results for patients to apply HBOT as early as possible in most situations. However, the hyperbaric oxygen toxicity cannot be ignored. The strategy of HBOT should be prudently drawn up to assure safety and effectivity, and sometimes the nutritional antioxidant supplementation could be combined with HBOT to avoid oxygen toxicity. Even though HBOT could only treat 
HAT clinically at present, we believe that it would be the most promising adjuvant therapy for other liver diseases after in-depth studies.

\section{Abbreviations}

ALF: acute liver failure; APAP: acetaminophen; ATA: atmosphere absolute; BDL: bile duct ligation; DEN: dimethylnitrosamine; GalN: galactosamine; GSH: glutathione; HAT: hepatic artery thrombosis; HBOT: hyperbaric oxygen therapy; HCC: hepatocellular carcinoma; IPRI: ischemia, preservation and reperfusion injury; I/R: ischemia-reperfusion; LPS: lipopolysaccharide; MDA: malondialdehyde; MPO: myeloperoxidase; MPT: mitochondrial permeability transition; MSG: monosodium glutamate; NAC: $\mathrm{N}$-acetylcysteine; NASH: nonalcoholic steatohepatitis; NO: nitric oxide; OLT: orthotopic liver transplantation; $\mathrm{PHx}$ : partial hepatectomy; RCR: respiratory control rate; SOD: superoxide dismutase; TAA: thioacetamide.

\section{Acknowledgements}

This work was supported by the Natural Science Foundation of Zhejiang Province (LGF18H170002) to ZB Chen, the Program of Chinese Medical Science of Zhejiang Province (2017ZK1003) to ZB Chen, and the Clinical Science Foundation of Zhejiang Medical Association to Y Sun (2017ZYC-A15).

\section{Competing Interests}

The authors have declared that no competing interest exists.

\section{References}

1. Savage S. New medical therapy: hyperbarics. Tennessee medicine : journal of the Tennessee Medical Association. 2010; 103: 39-40.

2. Tibbles PM, Edelsberg JS. Hyperbaric-oxygen therapy. The New England journal of medicine. 1996; 334: 1642-8.

3. Zarrinpar A, Busuttil RW. Liver transplantation: past, present and future. Nature reviews Gastroenterology \& hepatology. 2013; 10: 434-40.

4. Muralidharan V, Christophi C. Hyperbaric oxygen therapy and liver transplantation. HPB: the official journal of the International Hepato Pancreato Biliary Association. 2007; 9: 174-82.

5. Lv H, Han $\mathrm{CH}$, Sun XJ, Liu WW. Application of hyperbaric oxygen in liver transplantation. Medical gas research. 2016; 6: 212-8.

6. Macdougall JD, Couplan RE. Organ culture under hyperbaric oxygen. Experimental cell research. 1967; 45: 385-98.

7. Slapak M, Wigmore RA, MacLean LD. Twenty-four hour liver preservation by the use of continuous pulsatile perfusion and hyperbaric oxygen. Transplantation. 1967; 5(Suppl):1154-8.

8. Spilg H, Uys CJ, Hickman R, Saunders SJ, Terblanche J. Twelve-hour liver preservation in the pig using hypothermia and hyperbaric oxygen. The British journal of surgery. 1972; 59: 273-6.

9. Ijichi $\mathrm{H}$, Taketomi A, Soejima $\mathrm{Y}$, Yoshizumi T, Uchiyama H, Shimada M, et al. Effect of hyperbaric oxygen on cold storage of the liver in rats. Liver international: official journal of the International Association for the Study of the Liver. 2006; 26: 248-53.

10. Tran NQ, Malcontenti-Wilson C, Hammoud S, Millar I, Christophi C, Muralidharan V. Hyperbaric oxygen therapy reduces the severity of ischaemia, preservation and reperfusion injury in a rat model of liver transplantation. HPB: the official journal of the International Hepato Pancreato Biliary Association. 2012; 14: 103-14.

11. Kihara K, Ueno S, Sakoda M, Aikou T. Effects of hyperbaric oxygen exposure on experimental hepatic ischemia reperfusion injury: relationship between its timing and neutrophil sequestration. Liver transplantation: official publication of the American Association for the Study of Liver Diseases and the International Liver Transplantation Society. 2005; 11: 1574-80.

12. Silveira MR, Margarido MR, Vanni JC, Nejo Junior R, Castro ESO. Effects of hyperbaric oxygen therapy on the liver after injury caused by the hepatic ischemia-reperfusion process. Acta cirurgica brasileira. 2014; 29 Suppl 1: 29-33.

13. Chaves JC, Fagundes DJ, Simoes Mde J, Bertoletto PR, Oshima CT, Taha MO, et al. Hyperbaric oxygen therapy protects the liver from apoptosis caused by ischemia-reperfusion injury in rats. Microsurgery. 2009; 29: 578-83.

14. Chaves JC, Neto FS, Ikejiri AT, Bertoletto PR, Teruya R, Santos Simoes R, et al. Period of Hyperbaric Oxygen Delivery Leads to Different Degrees of Hepatic Ischemia/Reperfusion Injury in Rats. Transplantation proceedings. 2016; 48: 516-20.

15. Margarido MR, Silveira MR, Vanni JC, Feres O, Castro ESO. Hyperoxic preconditioning in partial liver ischemia. Acta cirurgica brasileira. 2014; 29 Suppl 1: 19-23

16. Yu SY, Chiu JH, Yang SD, Yu HY, Hsieh CC, Chen PJ, et al. Preconditioned hyperbaric oxygenation protects the liver against ischemia-reperfusion injury in rats. The Journal of surgical research. 2005; 128: 28-36.

17. Caldeira DE, Silveira MR, Margarido MR, Vanni JC, Feres O, Silva OC. Effect of hyperbaric hepatic hyperoxia on the liver of rats submitted to intermittent ischemia/reperfusion injury. Acta cirurgica brasileira. 2014; 29 Suppl 1: 24-8.

18. Losada DM, Jordani ME, Jordani MC, Piccinato MA, Fina CF, Feres O, et al. Should preconditioning hyperbaric oxygenation protect the liver against ischemia-reperfusion injury? An experimental study in a rat model. Transplantation proceedings. 2014; 46: 56-62.

19. Losada DM, Chies AB, Feres O, Chaib E, D'Albuquerque LA, Castro-e-Silva O. Effects of hyperbaric oxygen therapy as hepatic preconditioning in rats submitted to hepatic ischemia/reperfusion injury. Acta cirurgica brasileira. 2014; 29 Suppl 2: 61-6.

20. Liu Y, Sun XJ, Liu J, Kang ZM, Deng XM. Heme oxygenase-1 could mediate the protective effects of hyperbaric oxygen preconditioning against hepatic ischemia-reperfusion injury in rats. Clinical and experimental pharmacology \& physiology. 2011; 38: 675-82.

21. Lima CX, Sanches MD, Rezende Neto JB, Silva RC, Teixeira MM, Souza Dda G, et al. Hyperbaric oxygen therapy aggravates liver reperfusion injury in rats. Acta cirurgica brasileira. 2008; 23: 315-21.

22. Caldeira DE, Souza ME, Gomes MC, Picinato MA, Fina CF, Feres O, et al. Effects of hyperbaric oxygen (HBO), as pre-conditioning in liver of rats submitted to periodic liver ischemia/reperfusion. Acta cirurgica brasileira. 2013; 28 Suppl 1: 66-71.

23. Ponikvar R, Buturovic J, Cizman M, Mekjavic I, Kandus A, Premru V, et al. Hyperbaric oxygenation, plasma exchange, and hemodialysis for treatment of acute liver failure in a 3-year-old child. Artificial organs. 1998; 22: 952-7.

24. Truss CD, Killenberg PG. Treatment of carbon tetrachloride poisoning with hyperbaric oxygen. Gastroenterology. 1982; 82: 767-9.

25. Pratilas V, Pratila MG, Bramis J, Smith H. The hepatoprotective effect of oxygen during halothane anesthesia. Anesthesia and analgesia. 1978; 57: 481-5.

26. Bernacchi A, Myers R, Trump BF, Marzella L. Protection of hepatocytes with hyperoxia against carbon tetrachloride-induced injury. Toxicologic pathology. 1984; 12: 315-23.

27. Burk RF, Reiter R, Lane JM. Hyperbaric oxygen protection against carbon tetrachloride hepatotoxicity in the rat. Association with altered metabolism. Gastroenterology. 1986; 90: 812-8.

28. Burk RF, Lane JM, Patel K. Relationship of oxygen and glutathione in protection against carbon tetrachloride-induced hepatic microsomal lipid peroxidation and covalent binding in the rat. Rationale for the use of hyperbaric oxygen to treat carbon tetrachloride ingestion. The Journal of clinical investigation. 1984; 74: 1996-2001.

29. Marzella L, Muhvich K, Myers RA. Effect of hyperoxia on liver necrosis induced by hepatotoxins. Virchows Archiv B, Cell pathology including molecular pathology. 1986; 51: 497-507.

30. Cuzzocrea S, Imperatore F, Costantino G, Luongo C, Mazzon E, Scafuro MA et al. Role of hyperbaric oxygen exposure in reduction of lipid peroxidation and in multiple organ failure induced by zymosan administration in the rat. Shock. 2000; 13: 197-203.

31. Rinaldi B, Cuzzocrea S, Donniacuo M, Capuano A, Di Palma D, Imperatore F, et al. Hyperbaric oxygen therapy reduces the toll-like receptor signaling pathway in multiple organ failures. Intensive care medicine. 2011; 37: 1110-9.

32. Salhanick SD, Belikoff B, Orlow D, Holt D, Reenstra W, Buras JA. Hyperbaric oxygen reduces acetaminophen toxicity and increases HIF-1alpha expression. Academic emergency medicine: official journal of the Society for Academic Emergency Medicine. 2006; 13: 707-14.

33. Taslipinar MY, Aydin I, Kaldirim U, Aydin FN, Agilli M, Eyi YE, et al. Hyperbaric oxygen treatment and $\mathrm{N}$-acetylcysteine ameliorate acetaminophen-induced liver injury in a rat model. Human \& experimental toxicology. 2013; 32: 1107-16.

34. Mori H, Shinohara H, Arakawa Y, Kanemura H, Ikemoto T, Imura S, et al. Beneficial effects of hyperbaric oxygen pretreatment on massive hepatectomy model in rats. Transplantation. 2007; 84: 1656-61.

35. Ijichi H, Taketomi A, Yoshizumi T, Uchiyama H, Yonemura Y, Soejima Y, et al. Hyperbaric oxygen induces vascular endothelial growth factor and reduces liver injury in regenerating rat liver after partial hepatectomy. Journal of hepatology. 2006; 45: 28-34.

36. Mizuguchi T, Oshima H, Imaizumi H, Kohara H, Kawamoto M, Nobuoka T, et al. Hyperbaric oxygen stimulates cell proliferation and normalizes multidrug 
resistance protein-2 protein localization in primary rat hepatocytes. Wound repair and regeneration: official publication of the Wound Healing Society [and] the European Tissue Repair Society. 2005; 13: 551-7.

37. Nagamine K, Kubota T, Togo S, Nagashima Y, Mori M, Shimada H. Beneficial effect of hyperbaric oxygen therapy on liver regeneration after $90 \%$ hepatectomy in rats. European surgical research Europaische chirurgische Forschung Recherches chirurgicales europeennes. 2004; 36: 350-6.

38. Ozden TA, Uzun H, Bohloli M, Toklu AS, Paksoy M, Simsek G, et al. The effects of hyperbaric oxygen treatment on oxidant and antioxidants levels during liver regeneration in rats. The Tohoku journal of experimental medicine. 2004; 203: 253-65.

39. Tolentino EC, Castro e Silva O, Zucoloto S, Souza ME, Gomes MC, Sankarankutty AK, et al. Effect of hyperbaric oxygen on liver regeneration in a rat model. Transplantation proceedings. 2006; 38: 1947-52.

40. Ren P, Kang Z, Gu G, Liu Y, Xu W, Tao H, et al. Hyperbaric oxygen preconditioning promotes angiogenesis in rat liver after partial hepatectomy. Life sciences. 2008; 83: 236-41.

41. Ticinovic-Kurir T, Cikes-Culic V, Zemunik T, Grkovic I, Terzic J, Padovan M, et al. Immunohistochemical analysis of hepatic ganglioside distribution following a partial hepatectomy and exposure to different hyperbaric oxygen treatments. Acta histochemica. 2008; 110: 66-75.

42. Suehiro T, Shimura T, Okamura K, Okada T, Okada K, Hashimoto S, et al. The effect of hyperbaric oxygen treatment on postoperative morbidity of left lobe donor in living donor adult liver transplantation. Hepato-gastroenterology. 2008; 55: 1014-9.

43. Ayvaz S, Kanter M, Aksu B, Sahin SH, Uzun H, Erboga M, et al. The effects of hyperbaric oxygen application against cholestatic oxidative stress and hepatic damage after bile duct ligation in rats. The Journal of surgical research. 2013; 183: 146-55.

44. Terzioglu D, Uslu L, Simsek G, Atukeren P, Erman H, Gelisgen R, et al. The Effects of Hyperbaric Oxygen Treatment on Total Antioxidant Capacity and Prolidase Activity after Bile Duct Ligation in Rats. Journal of investigative surgery: the official journal of the Academy of Surgical Research. 2016: 1-7.

45. Tsuneyama K, Chen YC, Fujimoto M, Sasaki Y, Suzuki W, Shimada T, et al. Advantages and disadvantages of hyperbaric oxygen treatment in mice with obesity hyperlipidemia and steatohepatitis. TheScientificWorldJournal. 2011; 11: 2124-35.

46. Moen I, Stuhr LE. Hyperbaric oxygen therapy and cancer--a review. Targeted oncology. 2012; 7: 233-42

47. Ueno S, Sakoda M, Kurahara H, Iino S, Minami K, Ando K, et al. Safety and efficacy of early postoperative hyperbaric oxygen therapy with restriction of transfusions in patients with HCC who have undergone partial hepatectomy. Langenbeck's archives of surgery. 2011; 396: 99-106.

48. Daruwalla J, Christophi C. The effect of hyperbaric oxygen therapy on tumour growth in a mouse model of colorectal cancer liver metastases. European journal of cancer. 2006; 42: 3304-11.

49. Peng HS, Liao MB, Zhang MY, Xie Y, Xu L, Zhang YJ, et al. Synergistic inhibitory effect of hyperbaric oxygen combined with sorafenib on hepatoma cells. PloS one. 2014; 9: e100814.

50. Hill GB. Hyperbaric oxygen exposures for intrahepatic abscesses produced in mice by nonsporeforming anaerobic bacteria. Antimicrobial agents and chemotherapy. 1976; 9: 312-7.

51. Chang KY, Tsai PS, Huang TY, Wang TY, Yang S, Huang CJ. HO-1 mediates the effects of $\mathrm{HBO}$ pretreatment against sepsis. The Journal of surgical research. 2006; 136: 143-53.

52. Oter S, Edremitlioglu M, Korkmaz A, Coskun O, Kilic D, Kisa U, et al. Effects of hyperbaric oxygen treatment on liver functions, oxidative status and histology in septic rats. Intensive care medicine. 2005; 31: 1262-8.

53. Akin ML, Uluutku H, Erenoglu C, Ilicak EN, Elbuken E, Erdemoglu A, et al. Hyperbaric oxygen ameliorates bacterial translocation in rats with mechanical intestinal obstruction. Diseases of the colon and rectum. 2002; 45: 967-72.

54. Castro e Silva O, Sankarankutty AK, Martinelli AL, Souza FF, Teixeira AC, Feres $\mathrm{O}$, et al. Therapeutic effect of hyperbaric oxygen in hepatic artery thrombosis and functional cholestasis after orthotopic liver transplantation. Transplantation proceedings. 2006; 38: 1913-7.

55. Grover I, Conley L, Alzate G, Lavine J, Van Hoesen K, Khanna A. Hyperbaric oxygen therapy for hepatic artery thrombosis following liver transplantation: current concepts. Pediatric transplantation. 2006; 10: 234-9.

56. Dubost $\mathrm{T}$, Goubaux $\mathrm{B}$, Duhalde $\mathrm{M}$, Raucoules-Aime $\mathrm{M}$, Wolkiewiez J, Gugenheim J. Use of hyperbaric oxygen for hepatic artery thrombosis following adult orthotopic liver transplantation. European journal of anaesthesiology. 2002; 19: 223-4

57. Mazariegos GV, O'Toole K, Mieles LA, Dvorchik I, Meza MP, Briassoulis G, et al. Hyperbaric oxygen therapy for hepatic artery thrombosis after liver transplantation in children. Liver transplantation and surgery: official publication of the American Association for the Study of Liver Diseases and the International Liver Transplantation Society. 1999; 5: 429-36.

58. Fuson RL, Saltzman HA, Smith WW, Whalen RE, Osterhout S, Parker RT. Clinical Hyperbaric Oxygenation with Severe Oxygen Toxicity: Report of a Case. The New England journal of medicine. 1965; 273: 415-9.

59. Nishiki K, Jamieson D, Oshino N, Chance B. Oxygen toxicity in the perfused rat liver and lung under hyperbaric conditions. The Biochemical journal. 1976; 160: $343-55$
60. Ying $\mathrm{W}$. NAD+/NADH and NADP+/NADPH in cellular functions and cell death: regulation and biological consequences. Antioxidants \& redox signaling. 2008; 10: 179-206.

61. Coupland RE, MacDougall JD, Myles WS, McCabe M. The effect of hyperbaric oxygen on mitochondrial enzymes, lipid peroxides and adenosine triphosphate-induced contraction of mitochondria in organ cultures and cell fractions of rat liver. The Journal of pathology. 1969; 97: 63-77.

62. Sanders AP, Hall IH, Woodhall B. Succinate: protective agent against hyperbaric oxygen toxicity. Science. 1965; 150: 1830-1.

63. Shang F, Gong X, Egtesadi S, Meydani M, Smith D, Perrone G, et al. Vitamin C prevents hyperbaric oxygen-induced growth retardation and lipid peroxidation and attenuates the oxidation-induced up-regulation of glutathione in guinea pigs. The Journal of nutritional biochemistry. 2002; 13: 307-13.

64. Patel V, Chivukula IV, Roy S, Khanna S, He G, Ojha N, et al. Oxygen: from the benefits of inducing VEGF expression to managing the risk of hyperbaric stress. Antioxidants \& redox signaling. 2005; 7: 1377-87. 